\title{
Proton-Selective ${ }^{17} \mathrm{O}-{ }^{-1} \mathrm{H}$ Distance Measurements in Fast Magic-Angle-Spinning Solid-State NMR Spectroscopy for the Determination of Hydrogen Bond Lengths
}

\author{
Andreas Brinkmann* and Arno P. M. Kentgens \\ Physical Chemistry/Solid State NMR, Institute for Molecules and Materials, Radboud University Nijmegen, \\ Toernooiveld 1, 6525 ED Nijmegen, The Netherlands \\ Received July 27, 2006; E-mail: a.brinkmann@science.ru.nl
}

We present a proton-selective method to determine ${ }^{17} \mathrm{O}-{ }^{1} \mathrm{H}$ distances in organic, biological and biomimetic materials by fast magic-angle-spinning (MAS) solid-state NMR spectroscopy. This method allows the determination of internuclear distances between specific $\left({ }^{17} \mathrm{O},{ }^{1} \mathrm{H}\right)$ spin pairs selectively. In this way medium-range ${ }^{17} \mathrm{O} \cdots{ }^{1} \mathrm{H}$ distances across hydrogen bonds can be estimated in the presence of short-range ${ }^{17} \mathrm{O}-{ }^{1} \mathrm{H}$ contacts sharing the same ${ }^{17} \mathrm{O}$ site. The method employs the newly developed symmetry-based radiofrequency (rf) pulse sequence $\mathrm{SR} 4_{1}^{2}$ applied to the protons to achieve heteronuclear dipolar recoupling, while simultaneously decoupling the homonuclear proton dipolar interactions. Fast MAS $(50 \mathrm{kHz})$ and high static magnetic fields $(18.8 \mathrm{~T})$ achieve the required proton spectral resolution.

Hydrogen bonding is an essential component of the structure and functioning of bio(mimetic) materials. ${ }^{17} \mathrm{O}$ solid-state NMR has started to develop into a powerful tool to study hydrogen bonding in nucleic acids, amino acids, peptides, and proteins, ${ }^{2}$ as it gives direct access to one of the hydrogen-bonding partners in $\mathrm{X}-\mathrm{H} \cdots \mathrm{O}(\mathrm{X}=\mathrm{O}, \mathrm{N}, \mathrm{C})$ hydrogen bonds. So far, mainly the indirect correlation of ${ }^{17} \mathrm{O}$ chemical shifts and quadrupolar couplings has been used for this purpose. However, the most direct and accurate method to characterize these types of hydrogen bonds is to measure $\mathrm{O} \cdots \mathrm{H}$ distances across the hydrogen bonds by determining ${ }^{17} \mathrm{O}-{ }^{1} \mathrm{H}$ heteronuclear dipolar couplings.

Important examples of moderate hydrogen bonds ${ }^{1}$ in biological systems are $\mathrm{N}-\mathrm{H} \cdots \mathrm{O}=\mathrm{C}$ hydrogen bonds in $\beta$-sheets and $\alpha$-helices and $\mathrm{O}-\mathrm{H} \cdots \mathrm{O}$ hydrogen bonds in polysaccharides. $\mathrm{O} \cdots \mathrm{H}$ distances $d$ across moderate hydrogen bonds are in the medium-range order of $150-220 \mathrm{pm}$, hence significantly longer than $\mathrm{O}-\mathrm{H}$ interbond distances (98-99 pm). O-H groups can act simultaneously as proton donors and acceptors, for example, in the $\mathrm{H} \cdots \mathrm{O}-\mathrm{H} \cdots \mathrm{O}$ type hydrogen bonds in polysaccharides such as cellulose. ${ }^{3}$ In the crystal structure of L-tyrosine $\cdot \mathrm{HCl}$ the $\mathrm{O}^{\eta}-\mathrm{H}^{\eta}$ group acts as donor in the $\mathrm{O}^{\eta}-\mathrm{H}^{\eta} \cdots \mathrm{Cl}^{-}$hydrogen bond, and simultaneously the $\mathrm{O}^{\eta}$ acts as acceptor in the intermolecular $\mathrm{O}^{\prime \prime}-\mathrm{H}^{\prime \prime} \cdots \mathrm{O}^{\eta}$ hydrogen bond ${ }^{4}$ (shown in Figure 1).

The determination of dipolar couplings under MAS conditions requires the application of recoupling rf pulse sequences in order to suspend the MAS averaging of these couplings over a defined time interval. ${ }^{5}$ Heteronuclear dipolar recoupling sequences should ideally possess the following properties in order to allow accurate ${ }^{17} \mathrm{O}-{ }^{1} \mathrm{H}$ distance determination in biological and biomimetic solids: (i) The strong proton homonuclear dipolar couplings should be efficiently suppressed. (ii) The measurement of medium- and long-range ${ }^{17} \mathrm{O}-{ }^{1} \mathrm{H}$ distances requires the ability to determine weak dipolar couplings in the presence of stronger ones, that is, dipolar truncation should be absent. Hence, the recoupled heteronuclear dipolar interactions should commute for different spin pairs. (iii) The ${ }^{1} \mathrm{H}$ chemical shift anisotropy (CSA) should have no influence on the ${ }^{17} \mathrm{O}-{ }^{1} \mathrm{H}$ distance determination as the CSA of carboxyl and

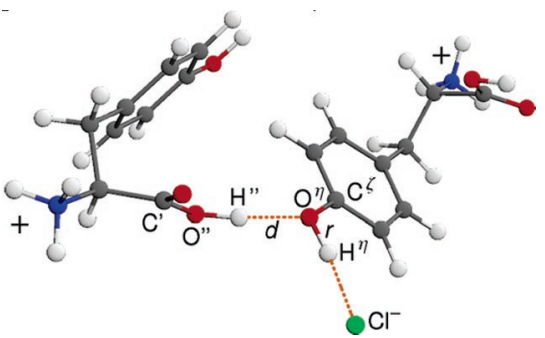

Figure 1. Two adjacent L-tyrosine cations and one chlorine anion in the neutron-diffraction-determined structure ${ }^{4}$ of L-tyrosine $\cdot \mathrm{HCl}$.

hydroxyl protons can be in the order of $20 \mathrm{ppm}$. (iv) The recoupling sequence should be solely applied to the ${ }^{1} \mathrm{H}$-spins, since the spin dynamics of quadrupolar nuclei in the presence of $\mathrm{rf}$ pulse sequences are generally complicated and site-dependent.

The rotational echo double resonance (REDOR) sequence 6 applied to the ${ }^{1} \mathrm{H}$ possesses the properties ii-iv; however, under fast MAS and the usual rf field limitations, substantial recoupling of the ${ }^{1} \mathrm{H}$ homonuclear dipolar interactions occurs. The symmetrybased $\mathrm{R} 18_{2}^{5}$ heteronuclear recoupling sequence fulfills the criteria $\mathrm{i}$ and iv and has been used to determine ${ }^{17} \mathrm{O}-{ }^{1} \mathrm{H}$ interbond distances. ${ }^{7}$ Recently, we presented supercycled symmetry-based $\mathrm{R} N_{n}^{v}$ pulse sequences achieving heteronuclear longitudinal twospin-order $\left(I_{z} S_{z}\right)$ recoupling that possess all properties i-iv. ${ }^{8}$ These sequences were used to determine the short-range $\mathrm{O}^{\eta}-\mathrm{H}^{\eta}$ interbond distance $r$ in $\mathrm{O}^{\eta}$-L-tyrosine $\cdot \mathrm{HCl}$ (Figure 1). Here we present a new sequence denoted $\mathrm{SR} 4{ }_{1}^{2}$ complying with all criteria $\mathrm{i}-\mathrm{iv}$ that provides an essential improvement over the existing sequences for estimating medium-range ${ }^{17} \mathrm{O} \cdot{ }^{1} \mathrm{H}$ distances, especially in the presence of short-range ${ }^{17} \mathrm{O}-{ }^{1} \mathrm{H}$ contacts. The sequence $\mathrm{SR} 4{ }_{1}^{2}$ is based on the basic rotor-synchronized sequence $\mathrm{R} 4_{1}^{2}=$ $180_{90} 180_{-90} 180_{90} 180_{-90}$ spanning exactly one rotational period. The notation $\beta_{\phi}$ denotes a pulse of flip angle $\beta$ and phase $\phi$ (both in degrees). A supercycle is constructed by adding a consecutive

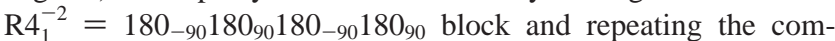
bined block $\mathrm{R} 4_{1}^{2} \mathrm{R} 4_{1}^{-2}$ three times with overall rf phase shifts $0^{\circ}$, $120^{\circ}$, and $240^{\circ}$, resulting in

$$
\mathrm{SR} 4_{1}^{2}=\left[\mathrm{R} 4_{1}^{2} \mathrm{R} 4_{1}^{-2}\right]_{0}\left[\mathrm{R} 4_{1}^{2} \mathrm{R} 4_{1}^{-2}\right]_{120}\left[\mathrm{R} 4_{1}^{2} \mathrm{R} 4_{1}^{-2}\right]_{240}
$$

(for a complete list of $\mathrm{rf}$ phases see the Supporting Information).

We employ the SR $4_{1}^{2}$ sequence in two different pulse schemes: The scheme in Figure 2a allows non-proton-selective ${ }^{17} \mathrm{O}-{ }^{1} \mathrm{H}$ distance determination, that is, the ${ }^{17} \mathrm{O}$ transverse magnetization is modulated by all ${ }^{17} \mathrm{O}-{ }^{1} \mathrm{H}$ dipolar interactions sharing the same ${ }^{17} \mathrm{O}$ site. The scheme in Figure $2 \mathrm{~b}$ allows determination of specific ${ }^{17} \mathrm{O}-$ ${ }^{1} \mathrm{H}$ distances by employing a selective $180^{\circ}$ Gaussian pulse on the protons following the concept of the selective REDOR sequence. ${ }^{9}$ The ${ }^{17} \mathrm{O}$ transverse magnetization is only modulated by those ${ }^{17} \mathrm{O}-$ ${ }^{1} \mathrm{H}$ dipolar interactions involving protons that are selectively inverted. ${ }^{9}$ 
a

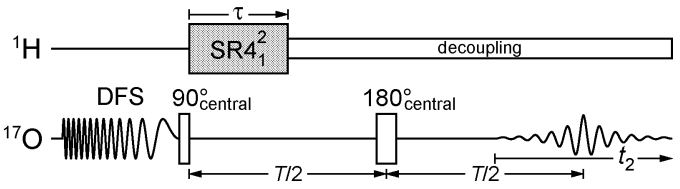

b

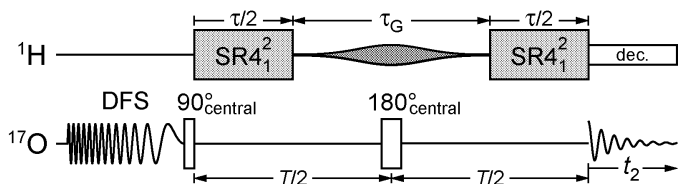

Figure 2. Pulse schemes for determining ${ }^{17} \mathrm{O}-{ }^{1} \mathrm{H}$ heteronuclear dipolar couplings. Both start by enhancing the ${ }^{17} \mathrm{O}$ central-transition population by a double-frequency sweep (DFS) ${ }^{10}$ followed by a Hahn echo sequence of duration $T$ employing ${ }^{17} \mathrm{O}$ central-transition-selective pulses ( $\mathrm{rf}$ field strength is small compared to the quadrupolar frequency). (a) Non-proton-selective experiment: For a time interval $\tau$ the ${ }^{17} \mathrm{O}$ central-transition transverse magnetization evolves under the $\mathrm{SR} 4_{1}^{2}$ heteronuclear recoupling sequence on the ${ }^{1} \mathrm{H}$. Subsequently, proton decoupling is applied and the ${ }^{17} \mathrm{O}$ spinecho is detected during $t_{2}$. The Hahn echo is time-constant as $\tau$ is incremented. (b) Proton-selective experiment: The SR $4_{1}^{2}$ sequence is split into two parts of equal duration $\tau / 2$ interrupted by a selective $180^{\circ}$ Gaussian pulse on the protons of duration $\tau_{\mathrm{G}}$ equal to an integer number of rotational periods. The Gaussian pulse is centered with respect to the $180^{\circ}$ pulse on the ${ }^{17} \mathrm{O}$. Half the ${ }^{17} \mathrm{O}$ spin-echo is detected during $t_{2}$. The total duration of the Hahn echo $T=\tau+\tau_{\mathrm{G}}$ increases as $\tau$ is incremented.
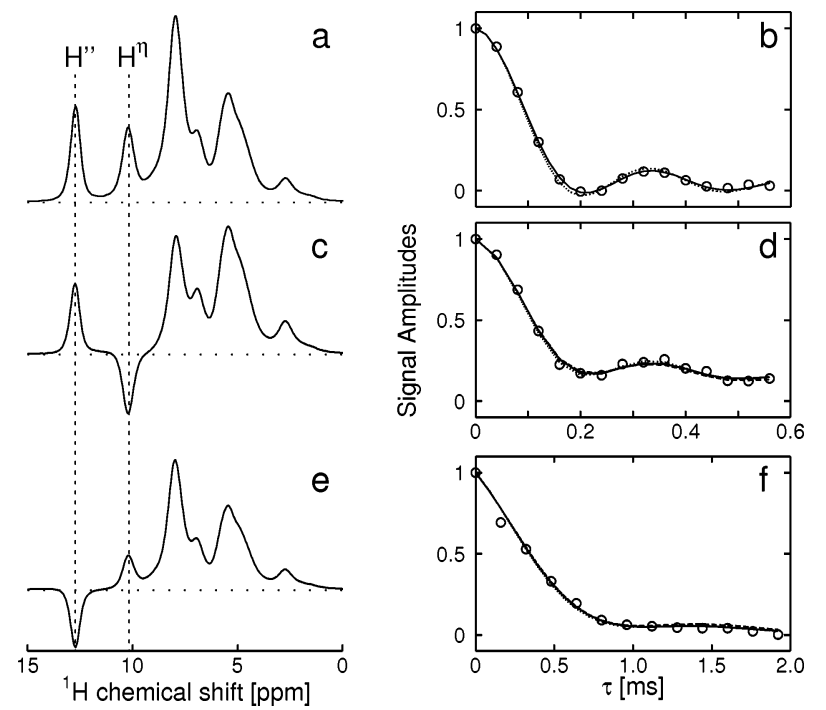

Figure 3. (a) Experimental ${ }^{1} \mathrm{H}$ spectrum of L-tyrosine $\cdot \mathrm{HCl}$ resulting from a Hahn echo sequence of $100 \mu$ s duration; (c) pulse sequence as in panel a preceded by a $180^{\circ}$ Gaussian pulse set to invert the ${ }^{1} \mathrm{H}^{\eta}$ longitudinal magnetization; (e) pulse sequence as in panel c except for the Gaussian pulse to invert the ${ }^{1} \mathrm{H}^{\prime \prime}$ longitudinal magnetization. (b, $\mathrm{d}$, and f) Normalized experimental integrals of the ${ }^{17} \mathrm{O}^{\eta}$ spectral peak as a function of the duration of the SR $4_{1}^{2}$ sequence: (b) using the pulse scheme in Figure 2a; (d) using the scheme in Figure $2 b$ with a ${ }^{1} \mathrm{H}^{\eta}$-selective Gaussian pulse; (f) using the scheme in Figure $2 b$ with a ${ }^{1} \mathrm{H}^{\prime \prime}$-selective Gaussian pulse. Solid lines represent the best-fit numerical two-spin simulations; dotted lines represent the average Hamiltonian calculations using the same fit parameters as for the solid black lines; dashed lines represent the best-fit numerical threespin simulations. All experiments were performed at a magnetic field of $18.8 \mathrm{~T}$ and a spinning frequency of $50 \mathrm{kHz}$ on a sample of $\left[35-40 \%{ }^{17} \mathrm{O}^{\eta}\right]-$ L-tyrosine $\cdot \mathrm{HCl}$. The ${ }^{1} \mathrm{H}$ rf field strength during the $\mathrm{SR} 4_{1}^{2}$ sequence was set to $100 \mathrm{kHz}$ using a two-dimensional nutation spectrum. The ${ }^{17} \mathrm{O}$ centraltransition-selective $180^{\circ}$ pulse length was set to one rotational period (20 $\mu \mathrm{s})$. The duration of the Gaussian pulse was set to 51 rotational periods $(1.02 \mathrm{~ms})$, and the frequency offset and $\mathrm{rf}$ amplitude was optimized to selectively invert ${ }^{1} \mathrm{H}^{\eta}$ and ${ }^{1} \mathrm{H}^{\prime \prime}$ longitudinal magnetization respectively, as shown in panels $\mathrm{c}$ and $\mathrm{e}$.

Experimental results obtained for ${ }^{17} \mathrm{O}^{\eta}$-L-tyrosine $\cdot \mathrm{HCl}$ using the pulse scheme in Figure 2a are shown in Figure 3b, where the solid line corresponds to the best-fit result of numerically exact two- spin $\left({ }^{17} \mathrm{O}^{\eta},{ }^{1} \mathrm{H}^{\eta}\right)$ simulations considering all relevant spin interactions. The best-fit value of the ${ }^{17} \mathrm{O}^{\eta}-{ }^{1} \mathrm{H}^{\eta}$ dipolar coupling constant is given by $14770 \pm 260 \mathrm{~Hz}(r=103 \pm 1 \mathrm{ppm})$ in agreement with our previous results. ${ }^{8}$ The dotted line is the result of average Hamiltonian calculations considering solely the heteronuclear dipolar coupling and using the same fitting parameters as for the solid black line. Figure 3 parts $\mathrm{d}$ and $\mathrm{f}$ show the experimental results obtained using the pulse scheme in Figure 2b, employing Gaussian pulses selective to the ${ }^{1} \mathrm{H}^{\eta}$ and ${ }^{1} \mathrm{H}^{\prime \prime}$ sites, respectively. The solid lines correspond to the best-fit results of numerical two-spin simulations. The best-fit value of the ${ }^{17} \mathrm{O}^{\eta}-{ }^{1} \mathrm{H}^{\eta}$ dipolar coupling constant corresponds to $14270 \pm 580 \mathrm{~Hz}$ (Figure 3d). This is in agreement within the error margins with the results obtained with the non-proton-selective scheme. The best-fit result of the ${ }^{17} \mathrm{O}^{\eta \cdots}{ }^{1} \mathrm{H}^{\prime \prime}$ dipolar coupling constant is given by $3130 \pm 660 \mathrm{~Hz}$ (Figure 3f). This corresponds to an intermolecular $\mathrm{O}^{\eta \cdots} \cdot \mathrm{H}^{\prime \prime}$ distance of $d=173$ $\pm 12 \mathrm{pm}$. Hence the solid-state NMR distance is about $8 \%$ larger than the value of $160.9 \mathrm{pm}$ determined by neutron diffraction. The accuracy is satisfactory considering the difficulty in measuring this type of distances by NMR. The dashed lines correspond to the bestfit results of numerical three-spin simulations where both the ${ }^{17} \mathrm{O}^{\eta}-$ ${ }^{1} \mathrm{H}^{\eta}$ and the ${ }^{17} \mathrm{O}^{\eta} \cdot{ }^{1} \mathrm{H}^{\prime \prime}$ dipolar couplings were varied on a twodimensional grid. The best-fit results for the ${ }^{17} \mathrm{O}^{\eta}-{ }^{1} \mathrm{H}^{\eta}$ and ${ }^{17} \mathrm{O}^{\eta} \cdot .{ }^{1} \mathrm{H}^{\prime \prime}$ dipolar coupling constants are given by 14200 and 3100 $\mathrm{Hz}$, respectively, which is in good agreement with the two-spin simulations.

In conclusion, we were able to determine selectively the mediumrange $\mathrm{O}^{\eta} \cdots \mathrm{H}^{\prime \prime}$ intermolecular hydrogen-bonding distance in the presence of the short-range $\mathrm{O}^{\eta}-\mathrm{H}^{\eta}$ interbond distance in ${ }^{17} \mathrm{O}^{\eta}$-Ltyrosine $\cdot \mathrm{HCl}$ employing the newly developed heteronuclear longitudinal two-spin-order recoupling sequence $\mathrm{SR} 4_{1}^{2}$. The presented pulse schemes are suitable in determining distances between protons and heteronuclei in general, irrespective of their spin quantum number. We anticipate numerous applications to characterize moderate hydrogen bonds in bio(mimetic) materials. To tackle larger systems involving spectral overlap, one can resort to selective ${ }^{17} \mathrm{O}$ labeling possibly combined with partial deuteration of the sample.

Acknowledgment. We thank Jan van Os, Gerrit Janssen, and Hans Janssen for experimental help and Ernst van Eck for discussions. A.B. has been supported by the VENI Grant 700.52.409 by The Netherlands Organization for Scientific Research (NWO).

Supporting Information Available: Further details on the experiments, simulations, and fitting procedure. This material is available free of charge via the Internet at http://pubs.acs.org.

\section{References}

(1) (a) Jeffrey, G. A. An Introduction to Hydrogen Bonding; Oxford University Press: New York, 1997. (b) Steiner, T. Angew. Chem. Int. Ed. 2002, 41, $48-76$.

(2) (a) Wu, G.; Dong, S.; Ida, R.; Reen, N. J. Am. Chem. Soc. 2002, 124 1768-1777. (b) Lemaître, V.; de Planque, M. R. R.; Howes, A. P.; Smith, M. E.; Dupree, R.; Watts, A. J. Am. Chem. Soc. 2004, 126, 15320-15321. (c) Hu, J.; Chekmenev, E. Y.; Gan, Z.; Gor'kov, P. L.; Saha, S.; Brey, W. W.; Cross, T. A. J. Am. Chem. Soc. 2005, 127, 11922-11923.

(3) Nishiyama, Y.; Langan, P.; Chanzy, H. J. Am. Chem. Soc. 2002, 124 9074-9082.

(4) Frey, M. N.; Koetzle, T. F.; Lehmann, M. S.; Hamilton, W. C. J. Chem. Phys. 1973, 58, 2547-2556.

(5) (a) Bennett, A. E.; Griffin, R. G.; Vega, S. NMR: Basic Princ. Prog. 1994, 33, 1-77. (b) Dusold, S.; Sebald, A. Ann. Rep. NMR Spectrosc. 2000, 41, 185-264. (c) Levitt, M. H. In Encyclopaedia of Nuclear Magnetic Resonance; Grant, D. M., Harris, R. K., Eds.; Wiley: Chichester 2002; Vol. 9, pp 165-196.

(6) Gullion, T.; Schaefer, J. J. Magn. Reson. 1989, 81, 196-200.

(7) van Beek, J. D.; Dupree, R.; Levitt, M. H. J. Magn. Reson. 2006, 179 , $38-48$.

(8) Brinkmann, A; Kentgens, A. P. M. J. Phys. Chem. B 2006, 110, 1608916101.

(9) Jaroniec, C. P.; Tounge, B. A.; Herzfeld, J.; Griffin, R. G. J. Am. Chem. Soc. 2001, 123, 3507-3519.

(10) Kentgens, A. P. M.; Verhagen, R. Chem. Phys. Lett. 1999, 300, 435-443. JA065415K 